\title{
Hybrid Management of Acute Portal Vein Thrombosis Complicated by Mesenteric Ischemia
}

\author{
Sayuri P Jinadasa', Mira Ghneim'1, Brittany O Aicher², Rishi Kundi', \\ John Karwowski ${ }^{3}$, Jose J Diaz' ${ }^{1}$ and Joseph J DuBose ${ }^{1}$ \\ 'R Adams Cowley Shock Trauma Center, University of Maryland Medical System, Baltimore, MD, USA \\ ${ }^{2}$ Division of General Surgery, University of Maryland Medical System, Baltimore, MD, USA \\ ${ }^{3}$ Division of Vascular Surgery, University of Maryland Medical System, Baltimore, MD, USA
}

\begin{abstract}
Acute portal vein thrombosis complicated by mesenteric ischemia requires emergent treatment to address the compromised bowel as well as the portal vein thrombus. We report a novel hybrid approach to managing this disease process. The procedure we discuss entails exploratory laparotomy and small bowel resection by the acute care emergency surgery team. Following this, the vascular surgery team performs a portal venogram through a branch mesenteric vein accessed through the laparotomy incision and then places a thrombolysis catheter. This technique and approach allows us to provide initial management efficiently and effectively under one operation.
\end{abstract}

Keywords: Mesenteric Ischemia; Endovascular Surgery; Emergency General Surgery; Portal Vein Thrombosis

Received: 6 August 2021; Accepted: 1 September 2021

The prevalence of portal vein thrombosis is estimated to be approximately $1 \%$ in the general population, with cirrhosis and hepatic carcinoma being the highest risk factors [1]. Portal vein thrombosis is often an incidental finding [2]; however, complete acute portal venous thrombosis can lead to abdominal pain and ascites. Intestinal ischemia and necrosis are the most serious complications $[3,4]$. The prevalence of this complication is not known; however, cases are described in the literature [4-6].

The goal of treatment is recanalization of the portal vein and prevention of thrombus extension. In cases with no clinical consequences, systemic anticoagulation is the treatment of choice [2]. Percutaneous endovascular catheter-directed and indirect thrombolysis, thrombectomy, and agitation thrombolysis [3] via

\section{Corresponding author:}

Sayuri P Jinadasa, R Adams Cowley Shock Trauma Center University of Maryland Medical System, 22 South Greene Street, Room T4N24, Baltimore, MD 21201, USA.

Email: Sayuri.jinadasa@som.umaryland.edu

(C) 2021 CC BY 4.0 - in cooperation with Depts. of Cardiothoracic/ Vascular Surgery, General Surgery and Anesthesia, Örebro University Hospital and Örebro University, Sweden transjugular or transhepatic access [7-9] are techniques developed for treatment of acute symptomatic portal vein thrombosis.

Our vascular surgery and acute care emergency surgery services developed a feasible and repeatable intraoperative hybrid technique to manage acute complicated portal vein thrombosis (Figure 1). Preoperatively, the patient is anticoagulated with systemic heparin. Upon abdominal exploration, the nonviable intestine is identified and resected in standard fashion (Figure 2). Within the cut edge of the mesentery along the line of the small bowel resection, a small distal branch vein is identified and cannulated with a micro-puncture needle, and subsequently a 5-French sheath is placed and a mesenteric venogram is performed (Figures 3 and 4). In the case being presented, the venogram demonstrated occlusive thrombus within the portal vein. A thrombolysis catheter is placed into the region of heaviest thrombus burden. Any areas of intra-abdominal bleeding are addressed with electrocautery and packed to avoid further bleeding when tissue plasminogen activator (tPA) administration is initiated post-operatively. At the end of the operation, an ABThera dressing (3M/KCI, St. Paul, MN, USA) is placed to allow for open abdominal packing while incorporating the thrombolytic catheter in a secured fashion (Figure 5). 


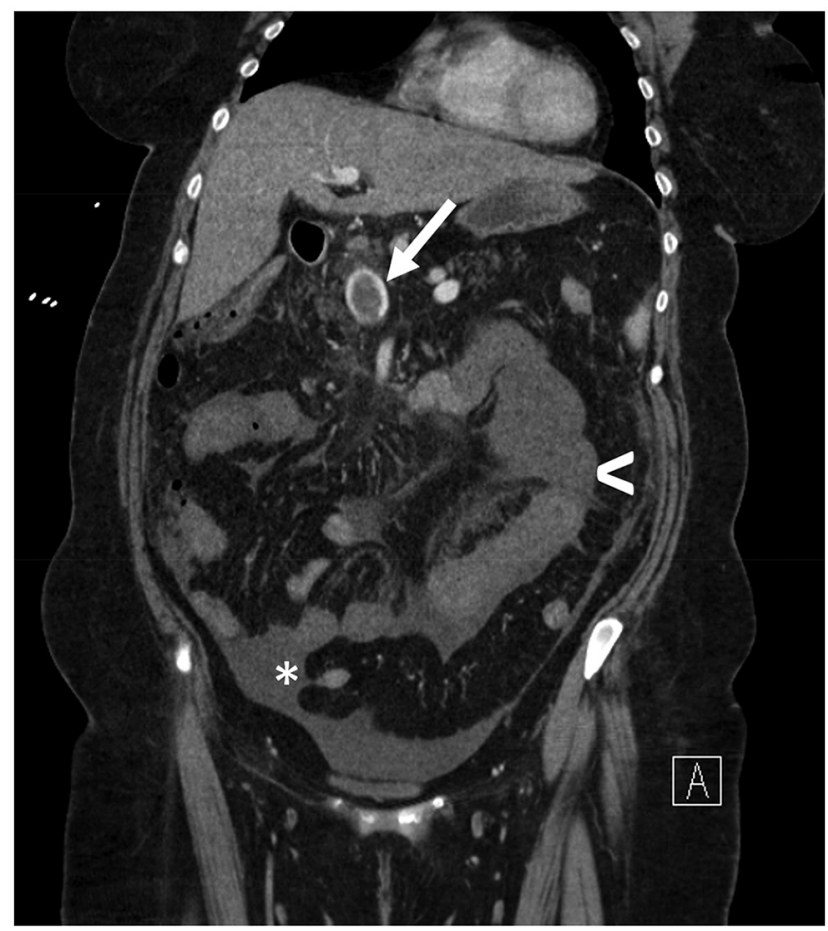

Figure 1 Computed tomography scan showing portal vein thrombus indicated by the arrow. Thickened small bowel with adjacent mesenteric edema is indicated by the arrowhead. The asterisk indicates free fluid.

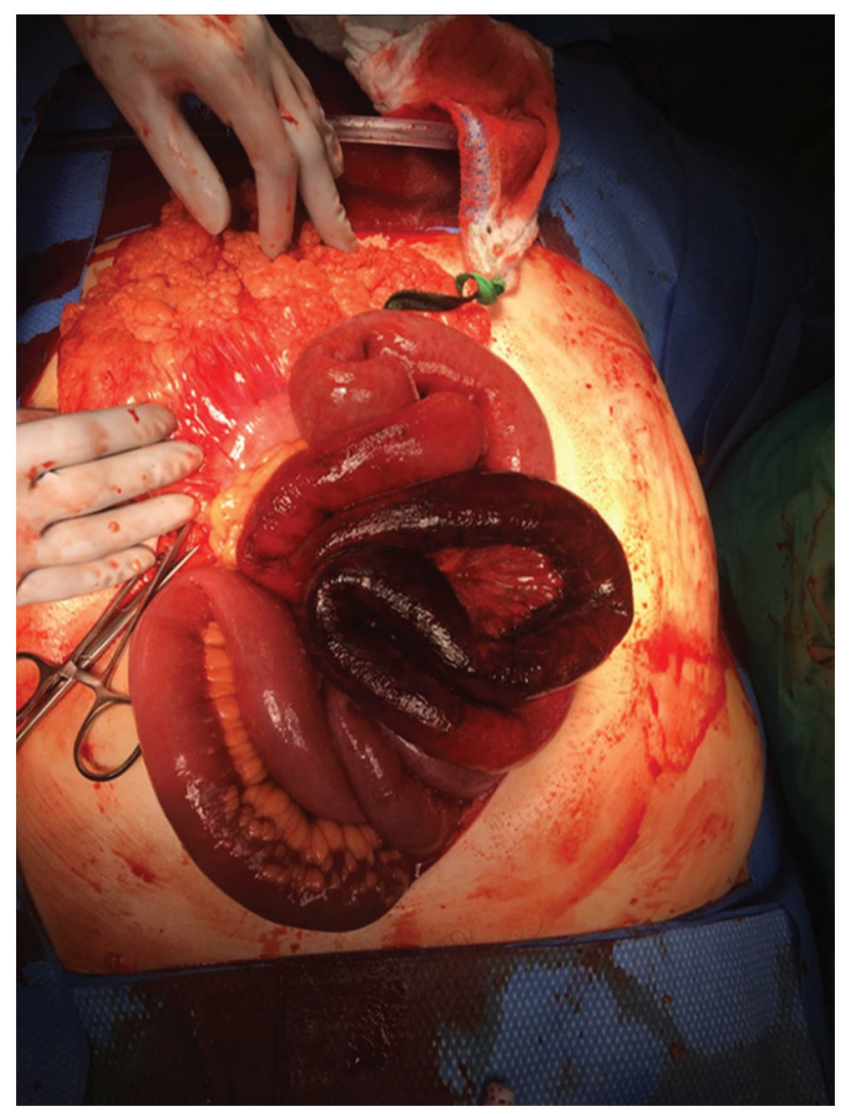

Figure 2 Nonviable jejunum identified during exploratory laparotomy.

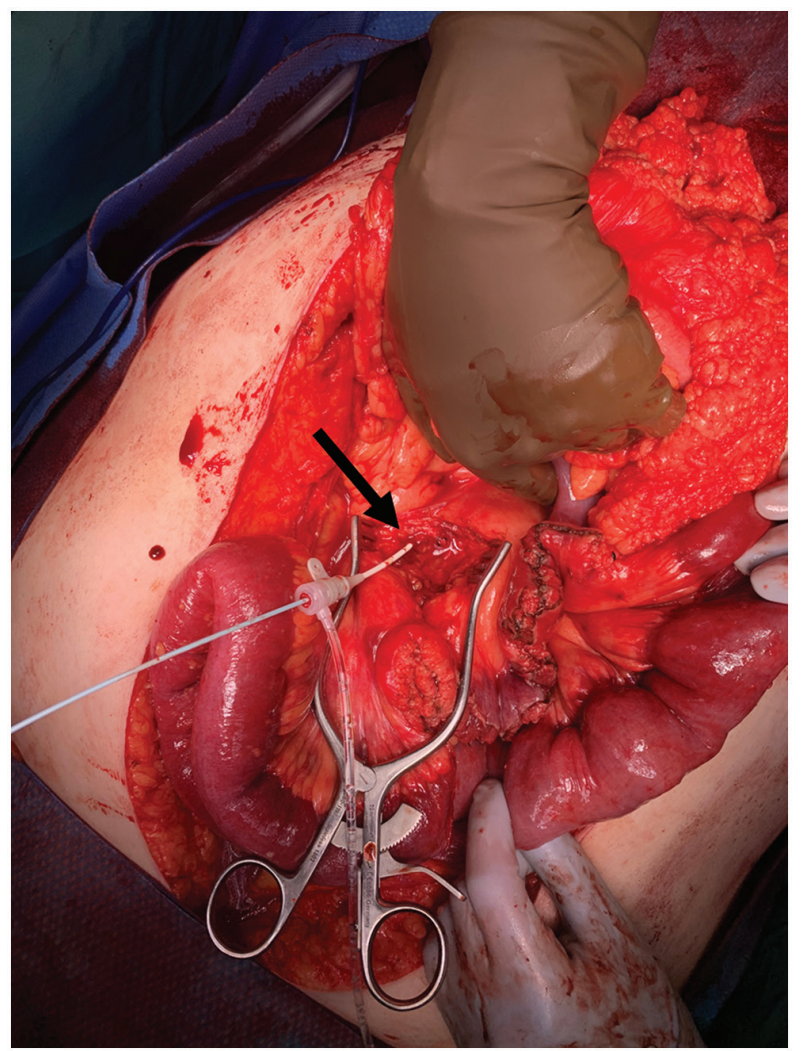

Figure 3 Venous system access with a 5-French sheath.

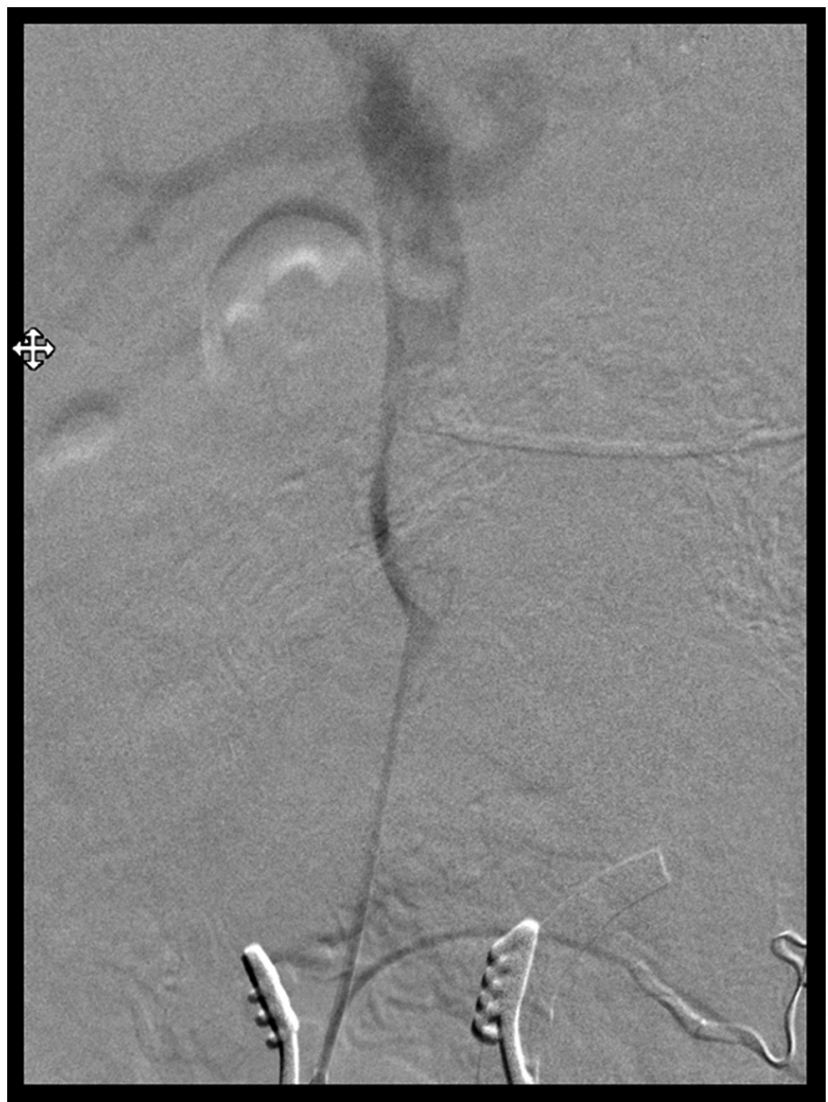

Figure 4 Pre-thrombolysis mesenteric venogram showing significant clot burden in the portal vein. 

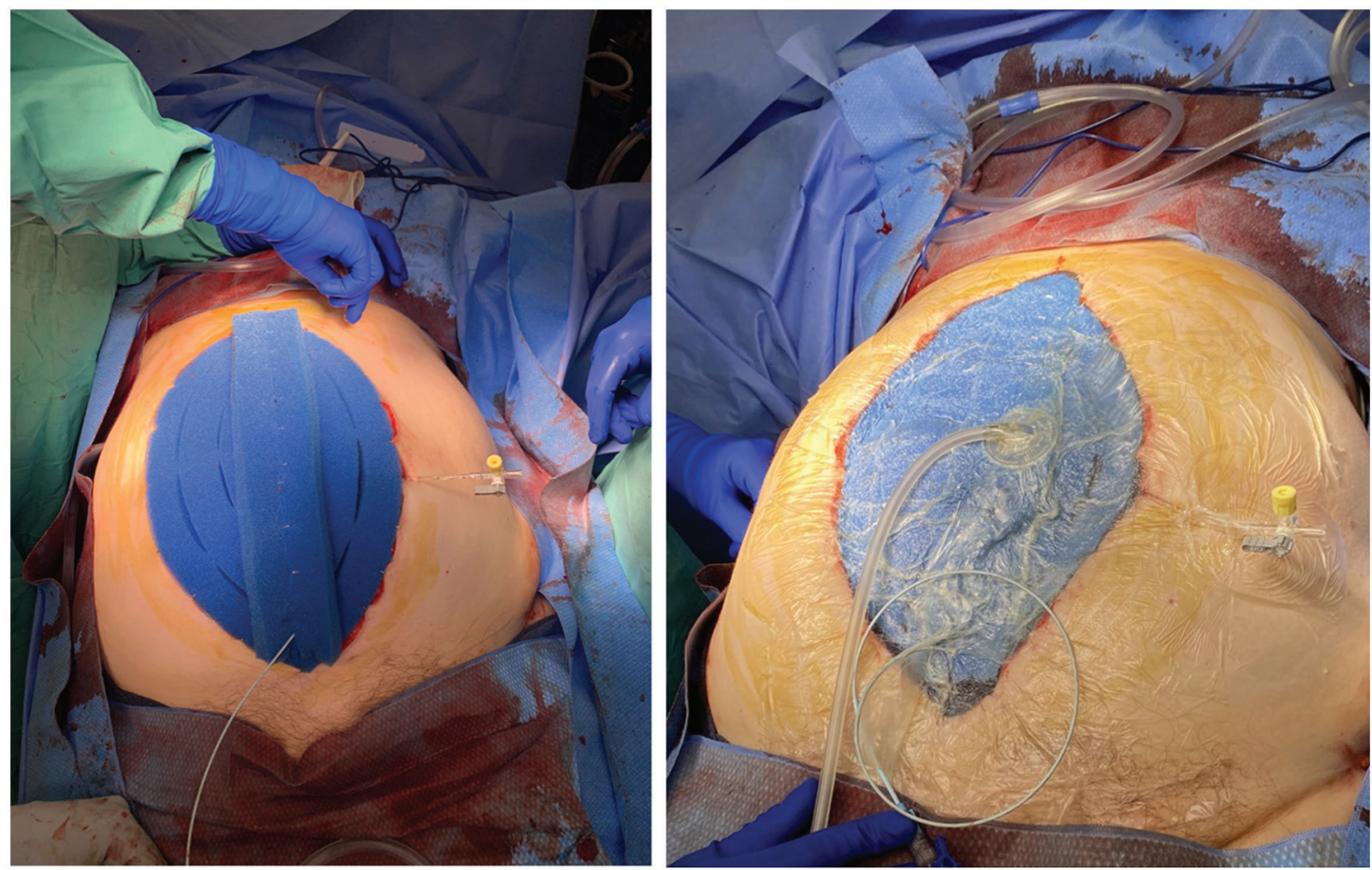

Figure 5 Placement of ABThera device in the OR with the sheath and thrombolysis catheter in place.

Postoperatively, the patient is monitored in the intensive care unit. tPA is administered initially via the catheter at a dose of $0.5 \mathrm{mg} / \mathrm{kg} / \mathrm{hr}$ and then titrated to maintain

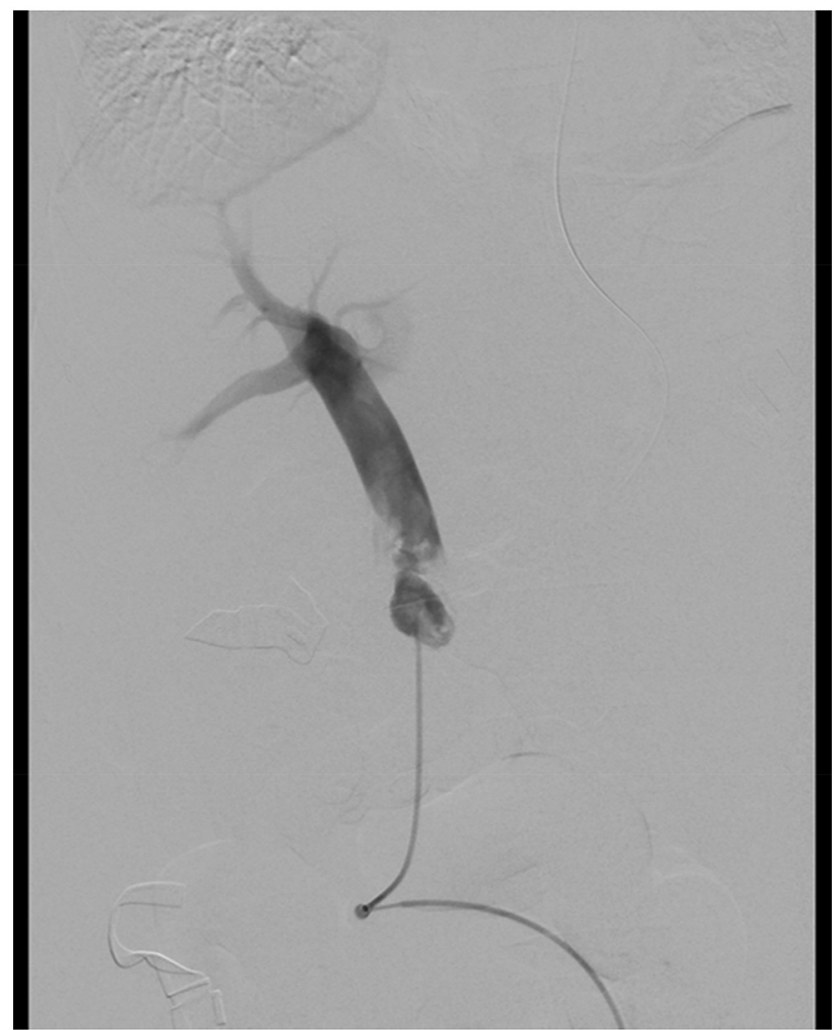

Figure 6 Post-thrombolysis mesenteric venogram showing reduction of clot burden. the fibrinogen level (checked every 6 hours) between 200 and $299 \mathrm{mg} / \mathrm{dL}$. Heparin is infused systemically to achieve a partial thromboplastin time level between 60 and 80 seconds as well as directly into the sheath at a non-titrated subtherapeutic dose of 500 units $/ \mathrm{hr}$.

Approximately 24 hours later, the patient is taken back to the operating room for re-evaluation of the intestine and repeat venogram (Figure 6). In the case of the presented patient, the repeat venogram showed a dramatic decrease of the portal vein clot burden and good outflow. The sheath is removed and the entry vein is suture ligated. Continuity of the intestine is re-established in standard fashion and the abdomen is closed if appropriate.

As described, this is a straightforward hybrid approach to manage portal vein thrombosis complicated by mesenteric ischemia that allows for efficient care of the patient, avoids the need for transhepatic cannulation for obtaining a venogram and placing a thrombolysis catheter, and obviates the need to obtain percutaneous venous access.

\section{Ethics Statement}

(1) All the authors mentioned in the manuscript have agreed to authorship, read and approved the manuscript, and given consent for submission and subsequent publication of the manuscript.

(2) The authors declare that they have read and abided by the JEVTM statement of ethical standards including rules of informed consent and ethical committee approval as stated in the article. 


\section{Conflicts of Interest}

The authors declare that they have no conflicts of interest.

\section{Funding}

The authors received no financial support for the research, authorship, and/or publication of this article.

\section{Author Contributions}

JJ DuBose, JK, and RK participated in the development of the technique. SPJ participated in the literature review. SPJ, JJ DuBose, BOA, and MG participated in the drafting of the article. SPJ, JJ Diaz, BOA, MG, RK, JJ Dubose and JK participated in the critical revision of the article. SPJ, JJ Diaz, MG and JJ Dubose participated in the approval of the version being submitted. All authors have contributed to the writing and editing of this manuscript.

\section{REFERENCES}

[1] Ogren M, Bergqvist D, Björck M, Acosta S, Eriksson H, Sternby NH. Portal vein thrombosis: prevalence, patient characteristics and lifetime risk: a population study based on 23,796 consecutive autopsies. World J Gastroenterol. 2006;12(13):2115-9.

[2] Rodrigues SG, Maurer MH, Baumgartner I, De Gottardi A, Berzigotti A. Imaging and minimally invasive endovascular therapy in the management of portal vein thrombosis. Abdom Radiol (NY). 2018;43(8):1931-46.
[3] Wang CY, Wei LQ, Niu HZ, Gao WQ, Wang T, Chen SJ. Agitation thrombolysis combined with catheter-directed thrombolysis for the treatment of non-cirrhotic acute portal vein thrombosis. World J Gastroenterol. 2018; 24(39):4482-8.

[4] Burch J, Enofe I. Acute mesenteric ischaemia secondary to portal, splenic and superior mesenteric vein thrombosis. BMJ Case Rep. 2019;12(9):e230145.

[5] Chen AZL, Allaway MGR, Al-Asady R, Richardson A. Management of acute extensive portal vein thrombosis: the complexity and morbidity. BMJ Case Rep. 2020; 13(6):e234282.

[6] Osti NP, Sah DN, Bhandari RS. Successful medical management of acute mesenteric ischemia due to superior mesenteric and portal vein thrombosis in a 27-year-old man with protein $S$ deficiency: a case report. J Med Case Rep. 2017;11(1):315.

[7] Chamarthy MR, Anderson ME, Pillai AK, Kalva SP. Thrombolysis and transjugular intrahepatic portosystemic shunt creation for acute and subacute portal vein thrombosis. Tech Vasc Interv Radiol. 2016;19(1): 42-51.

[8] Jiang TT, Luo XP, Sun JM, Gao J. Clinical outcomes of transcatheter selective superior mesenteric artery urokinase infusion therapy vs transjugular intrahepatic portosystemic shunt in patients with cirrhosis and acute portal vein thrombosis. World J Gastroenterol. 2017; 23(41):7470-7.

[9] Cai G, Li C, Hua Z, et al. Angiojet aspiration thrombectomy combined with transcatheter thrombolysis in treatment of acute portal venous systemic thrombosis. Ann Vasc Surg. 2020;66:362-9. 